\title{
Mechanical testing of a device for subcutaneous internal anterior pelvic ring fixation versus external pelvic ring fixation
}

\author{
Georg Osterhoff ${ }^{1 *}$, Simon Tiziani ${ }^{1}$, Stephen J Ferguson ${ }^{2}$, Gregor Spreiter $^{2}$, Max J Scheyerer ${ }^{1}$, Gian-Leza Spinas ${ }^{1}$, \\ Guido A Wanner ${ }^{1}$, Hans-Peter Simmen ${ }^{1}$ and Clément ML Werner ${ }^{1}$
}

\begin{abstract}
Background: Although useful in the emergency treatment of pelvic ring injuries, external fixation is associated with pin tract infections, the patient's limited mobility and a restricted surgical accessibility to the lower abdomen. In this study, the mechanical stability of a subcutaneous internal anterior fixation (SIAF) system is investigated.

Methods: A standard external fixation and a SIAF system were tested on pairs of Polyoxymethylene testing cylinders using a universal testing machine. Each specimen was subjected to a total of 2000 consecutive cyclic loadings at $1 \mathrm{~Hz}$ with sinusoidal lateral compression/distraction $(+/-50 \mathrm{~N})$ and torque $(+/-0.5 \mathrm{Nm})$ loading alternating every 200 cycles. Translational and rotational stiffness were determined at 100, 300, 500, 700 and 900 cycles.

Results: There was no significant difference in translational stiffness between the SIAF and the standard external fixation when compared at $500(p=.089), 700(p=.081)$, and $900(p=.266)$ cycles. Rotational stiffness observed for the SIAF was about 50 percent higher than the standard external fixation at $300(p=.005), 500(p=.020)$, and $900(p=.005)$ cycles. No loosening or failure of the rod-pin/rod-screw interfaces was seen.
\end{abstract}

Conclusions: In comparison with the standard external fixation system, the tested device for subcutaneous internal anterior fixation (SIAF) in vitro has similar translational and superior rotational stiffness.

Keywords: Pelvic fracture, Pelvic ring injury, Internal fixation, External fixation, Subcutaneous internal anterior fixation

\section{Background}

External fixation has been an established technique in the emergency treatment of pelvic ring injuries [1,2]. With external fixation, reduction of the inner pelvic diameter can be achieved within minutes, thereby limiting venous haemorrhage into the lesser pelvis [3]. Besides this direct effect on the extent of blood loss in the acute situation, external fixation reduces motion between the pelvic fragments and thus helps to maintain haemostasis during the first days after the trauma $[4,5]$.

It has been shown that supra-acetabular positioning of the pins leads to improved anchorage of the pins and a higher construct stability when compared to a pin placement into the iliac crest [6].

\footnotetext{
* Correspondence: georg.osterhoff@usz.ch

'Division of Trauma Surgery, University of Zurich, Raemistrasse 100, Zurich 8091, Switzerland

Full list of author information is available at the end of the article
}

Particularly for pelvic injuries with posterior instability, appropriate stability can only be achieved by combined anterior and posterior fixation or a sufficient posterior fixation alone $[7,8]$.

Thus, definitive internal pelvic stabilization is usually applied as soon as the patient's condition allows for more extensive surgery [9].

Besides usually serving only as a temporary stabilization, the use of external fixation of pelvic fractures is associated with further disadvantages. Pin tract infections are common and can occur in up to $50 \%$ of cases [10]. Rolling the patient side-to-side, sitting and lying in prone position is limited. Especially in patients with concomitant intra-abdominal injuries, where multiple revisions might be necessary, an anterior frame impairs the access to the lower abdomen. In addition, achieving stability by external fixation in severely obese patients with a large distance between the pelvis and the connecting rods 
is almost impossible, due to abundant abdominal soft tissue.

Therefore, a subcutaneous internal anterior screw-rod fixation has been suggested that can be applied within a comparable time to an external fixateur, while avoiding the disadvantages of the external implant [11]. This new technique has already been tested in several case series, where indeed very low rates of wound infections were observed when compared to those of external fixation. Yet, it was associated new complications like irritation of the lateral femoral cutaneous nerve or heterotopic ossifications [11-13].

With the development of minimal-invasive spinal instrumentation systems, there are new possibilities to perform this technique of subcutaneous internal anterior fixation (SIAF) in a less invasive fashion.

Little is known about the mechanical properties of such instrumentation. Failure to achieve stable fixation can result in the detachment of blood clots at the fracture site and therefore put the patient at risk for recurrent bleeding. As well, loosening of the rod-screw interface would require revision surgery.

The purpose of this study was to investigate the in vitro mechanical stability of a device for subcutaneous internal anterior fixation and compare it to a standard external fixation.

\section{Methods}

\section{Testing samples}

The experimental setup was designed to progressively measure the fatigue life or damage accumulation of the rod-pin/rod-screw interfaces. Two fixation techniques were tested on pairs of Polyoxymethylene testing cylinders with a diameter of $70 \mathrm{~mm}$ (Delrin, DuPont, Wilmington, DE, USA). These cylinders provided sound purchase of the pelvic pins and screws thereby simulating optimum anchorage in the pelvic bone.

In the group EXTERNAL $(\mathrm{n}=3)$, a standard external fixateur (Hoffmann II, Stryker, Kalamazoo, MI, USA) was mounted (Figure 1A) using two apex self-drilling pins (diameter $5 \mathrm{~mm}$ ) each anchored in one of on two separate testing cylinders. Angles and distances between the two pins were chosen in a way to reproduce the conditions as measured for the supra-acetabular channel on a pelvic model (Pelvis, Synbone, Malans, Switzerland) (Figure 1C). Two connecting carbon fiber rods (diameter $8 \mathrm{~mm}$ ) were attached using pin-to-rod couplings according to the manufacturer's instructions. Like in clinical routine, we aimed for torque forces exceeding that of a torque-limiter. Thus, all couplings were tightened with maximum effort by the same person (S. T.).

In the group SIAF $(\mathrm{n}=3)$, two 8 -mm-screws (Iliac Multiaxial Screw, Legacy, Medtronic, Minneapolis, MN, USA) were placed in two testing cylinders in exactly the same way as described for the supra-acetabular pins in the EXTERNAL group Figure 1B, D). The screws were connected by a contoured titanium rod (diameter 8.5; Longitude, Medtronic, Minneapolis, MN, USA). Couplings in the polyaxial screw heads were tightened by the same person (S. T.) with a torque screwdriver according to the manufacturer's instructions (Legacy, Medtronic, Minneapolis, MN, USA).

\section{Mechanical testing}

In patients, two basic loading scenarios are likely after provisional stabilization of pelvic ring fractures. First,

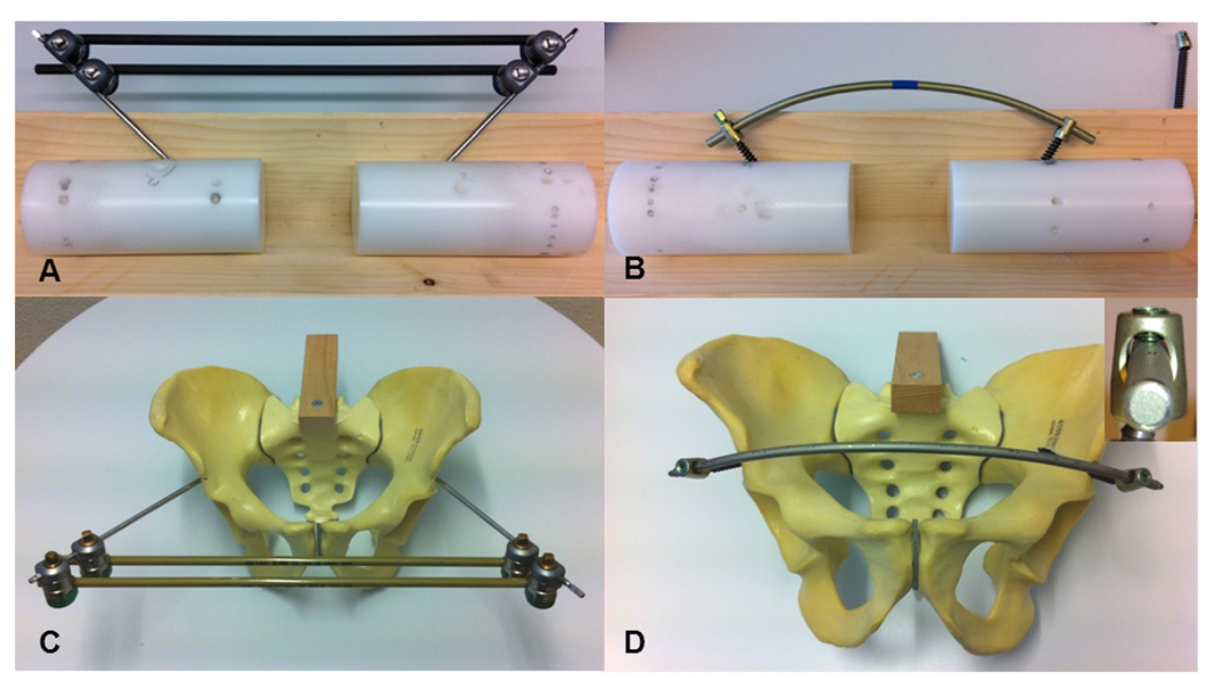

Figure 1 Testing samples. Reproducing angles and distances measured on similar pelvic models (C, D), a standard external fixateur (A) and a subcutaneous internal anterior fixation system (B) were mounted on pairs of Polyoxymethylene testing cylinders. The small image on the right shows a close-up of the coupling of the subcutaneous internal anterior fixation system. 
rolling the patient side-to-side is common (e.g. when changing bed-linen) which would result in lateral compression forces on the pelvic fixation construct in the transversal plane. Second, bending of the hip or transfer to a chair would result in torque forces in the sagittal plane.

Thus both fixation constructs were tested by alternating cycles of lateral compression/distraction (i.e. following the cylinder axis) and torque forces (i.e. counter-rotation of the cylinders about the central axis) using a universal testing machine (ElectroPulse E10000, Instron, High Wycombe, UK). The cylinder axes of the samples were aligned with the axis of the actuator using universal joints to eliminate confounding bending moments (Figure 2). After observing a displacement of clinical relevance (> $25 \mathrm{~mm})$ at $+/-50 \mathrm{~N}$, this load was chosen for the experimental setup.

Each specimen was subjected to total of 2000 consecutive cyclic loadings at $1 \mathrm{~Hz}$ with sinusoidal lateral compression/distraction $(+/-50 \mathrm{~N})$ and torque $(+/-0.5 \mathrm{Nm})$. One thousand cycles of rotation and 1000 cycles of compression/distraction with the mode of loading alternating every 200 cycles were applied.

During testing, loading forces and displacement were registered by a $+/-1000 \mathrm{~N}$ (compression/distraction forces) and a $25 \mathrm{Nm}$ (torque forces) load cell and translational and rotational stiffness were calculated. Failure resulting in immediate stop of testing included: displacement beyond the machine's range of motion $(+/-30 \mathrm{~mm}$

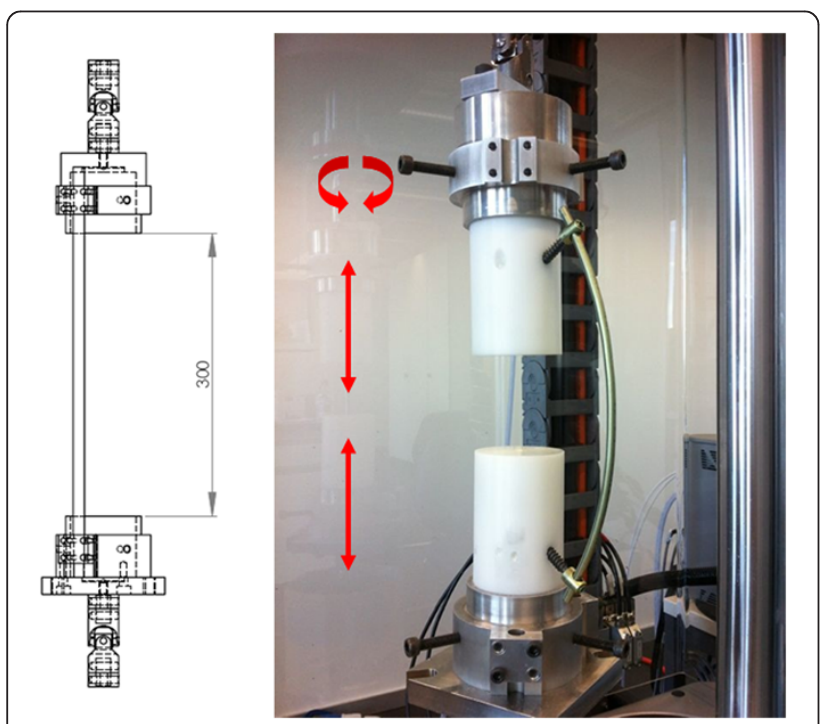

Figure 2 Experimental setup. On a universal testing machine, 2000 consecutive cyclic loadings were applied with sinusoidal lateral compression/distraction and torque loading alternating every 200 cycles. The cylinder axes of the samples were aligned with the axis of the actuator using universal joints to eliminate confounding bending moments (see dimensional sketch on the left). in the transversal plane, +/- 135 degrees rotation) or interface failure.

The following parameters were evaluated:

\section{Translational stiffness [N/mm]}

A measure of the extent to which the fixation system resisted deformation in response to a force simulating lateral compression of the pelvis. This value was determined at 100, 300, 500, 700 and 900 cycles.

\section{Rotational stiffness [Nm/degree]}

A measure of the extent to which the fixation system resisted deformation in response to a moment simulating torque of the pelvis. This value was determined at 100, 300, 500, 700 and 900 cycles.

\section{Statistical analysis}

After preliminary testing, a sample size calculation was performed using PS Power and Sample Size Calculations 3.0 (alpha error: 0.05) [14].

With an expected difference in means of $0.5 \mathrm{~N} / \mathrm{mm}$ and a standard deviation of $0.15 \mathrm{~N} / \mathrm{mm}$ for translational stiffness and an expected difference in means of $0.08 \mathrm{Nm} /$ degree and a standard deviation of $0.025 \mathrm{Nm} /$ degree for rotational stiffness the calculated number of samples to be able to reject the null hypothesis that the population means of the experimental and control groups are equal with a probability (power) of 0.8 was 3 per group.

After testing for normal distribution by a ShapiroWilk test, comparison of translational and rotational stiffness was done using a paired T-Test with SPSS for Windows V14.0 (SPSS, Chicago, Illinois, USA). Differences were considered significant for values of $p<0.05$.

\section{Results}

Translational stiffness

There was a slight but not significant difference, when tested in response to a force simulating lateral compression of the pelvis, between the stiffness observed for the internal anterior fixation (SIAF) and the standard external fixation (EXTERNAL) when compared at $500(\mathrm{p}=.089)$, $700(\mathrm{p}=.081)$, and $900(\mathrm{p}=.266)$ cycles. At $100(\mathrm{p}=.038)$ and $300(\mathrm{p}=.021)$ cycles, however, the translational stiffness was even slightly higher in the SIAF group (Figure 3).

\section{Rotational stiffness}

When tested in response to a force simulating torque of the pelvis, the stiffness observed for the subcutaneous internal anterior fixation (SIAF) was about 50 percent higher than the standard external fixation (EXTERNAL) at all times of measurement. This was statistically significant when compared at $300(\mathrm{p}=.005), 500(\mathrm{p}=.020)$, and $900(\mathrm{p}=.005)$ cycles. At $100(\mathrm{p}=.050)$ and 700 


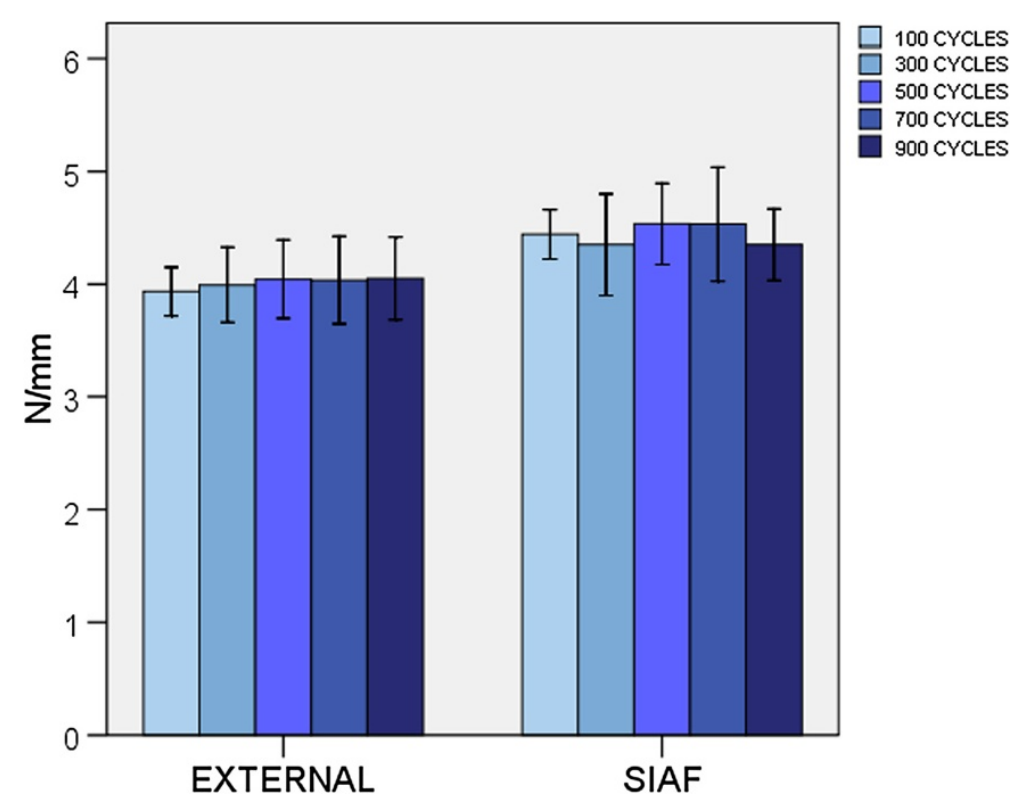

Figure 3 Translational stiffness. EXTERNAL: standard external fixation. SIAF: subcutaneous internal anterior fixation. Error bars represent 2 standard deviations.

$(\mathrm{p}=.050)$ cycles, the level of significance was not achieved (Figure 4).

Temporary deformation was solely caused by elastic bending of the rods, screws and pins, the rod-pin/rodscrew interfaces did not show any loosening or failure with the applied loads. Persistent plastic deformation was not seen.

\section{Discussion}

Supra-acetabular external fixation of pelvic ring injuries is an efficient tool to minimize blood loss in the acute situation and to maintain haemostasis during the first days after the trauma [1-6]. However, pin tract infections, the patients' limited mobility and a restricted surgical accessibility to the lower abdomen are noticeable

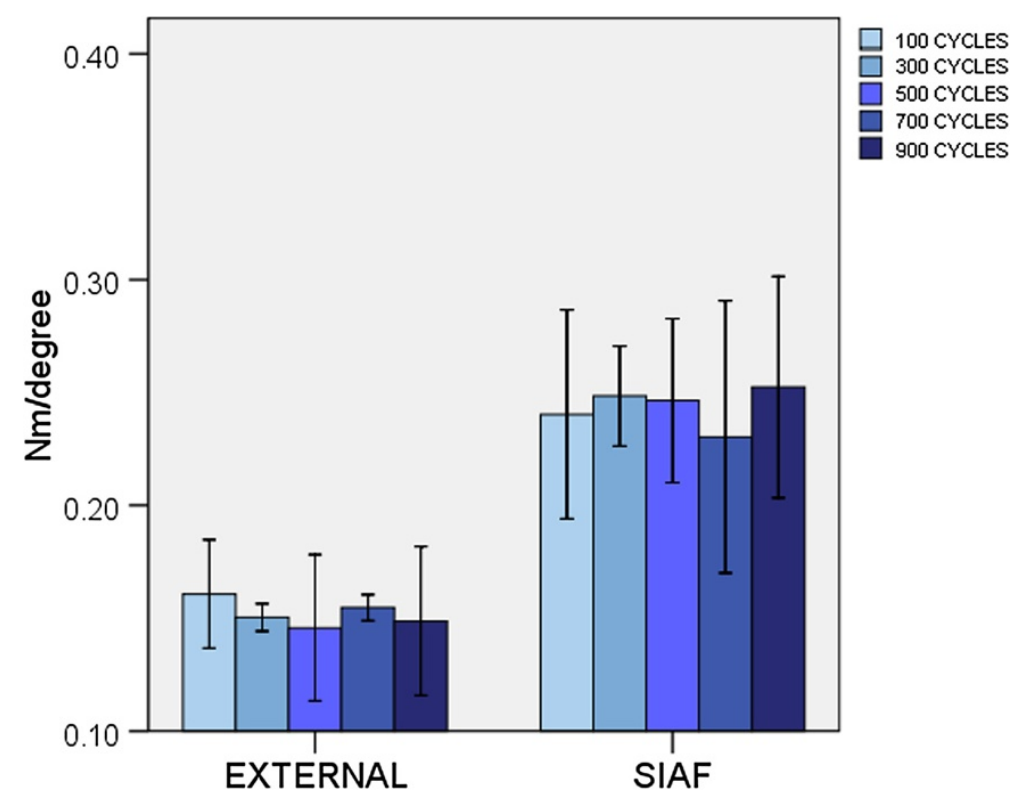

Figure 4 Rotational stiffness. EXTERNAL: standard external fixation. SIAF: subcutaneous internal anterior fixation. Error bars represent 2 standard deviations. 
disadvantages of this technique. In 2009, Kuttner et al. introduced the idea of a subcutaneous internal anterior fixation of the pelvis [11].

This study tested the in vitro mechanical stability of a subcutaneous internal anterior fixation system (SIAF) in comparison to a standard external fixation (EXTERNAL). We used an experimental setup with a focus on the rodpin/rod-screw interface, which was thought to be the weakest link within both fixation techniques and simulated forces resulting from side-to-side rolling of a patient (translational stiffness) and forces resulting from bending of the hip (rotational stiffness). The SIAF system showed a similar to slightly higher translational stiffness than the EXTERNAL system and a significantly higher rotational stiffness at up to 900 loading cycles. It is important to note that this combined implant-construction stiffness is a function of rod- and pin/screw stiffness, pin/screw-rod interface motility and the swinging distance of the implant over the bone surrogate. It is our conviction that this difference is largely caused by the large swinging distance of the pins over the bone in external fixation. This problem is partly eliminated by the positioning of the rod in SIAF.

Several case series using a subcutaneous internal anterior fixation showed good short-term results with only few complications related to implant failure [11-13]. Our in vitro data is consistent with these clinical experiences. In addition, there is one recent mechanical study comparing a similar subcutaneous anterior fixation technique with anterior external and plate fixation suggesting superior stiffness of the SIAF-like system [15]. The latter study, however, uses only one single connecting rod with the external fixation, which is known to have inferior mechanical stability [16] and therefore usually does not represent the clinical routine. In contrast to our study, a cyclic loading setup was not used by Vigdorchik et al. [15]. In most in vivo cases, however, postoperative fragment dislocation is not a result of a single trauma or force, but rather occurs due to repetitive motion (e.g. by nursing bedside assistance or physiotherapy) as is suggested by the typical appearance of symmetric aseptic loosening of the pins in many cases [10]. Therefore, cyclic loading seems to better approximate clinical loading scenarios.

To date, there exists no standard in mechanical testing of pelvic ring fixations. Generally, a cadaver or synthetic pelvis is tested with large variations in force vectors' direction, quantity and point of application [4,15,17-21]. Most experimental setups ignore forces resulting by acceleration when walking and ignore force vector realignment by abductor and adductor muscles [17,22,23].

There is also no gold standard for external fixation configurations in the treatment of pelvic ring injuries, even single-rod fixation has been described [15]. A double-rod configuration as used in our study, however, has been considered to provide sufficient stability [16].
The stability of pelvic fixation systems is defined by their stiffness and by possible failure of the rod-pin/rodscrew interfaces. As data for in vivo loading of the pelvic ring are limited, the test was designed to progressively measure the fatigue life or damage accumulation of these rod-pin/rod-screw interfaces [24]. A simplified setup was chosen with a focus on the stiffness of the implants themselves as well as on the specified interfaces. By choosing this test protocol, confounding factors were avoided, such as the quality of screw/pin anchorage, which depends on bone quality and surgical application. With the results of this study, no conclusions can be drawn on the implant's behaviour at the implant-boneinterface. It is possible that the increased stiffness of the SIAF system prevents loosening of bone anchorage, or also that it even promotes loosening by increased stresses at the implant-bone-interface [25]. The very low implant loosening rates in the few known clinical series using the SIAF [11-13] support the idea of improved anchorage - due to a higher stiffness and stability and due to the greater diameter of the SIAF screws.

All the latter clinical studies applied the SIAF in combination with posterior fixation. Future studies may further analyse the implant-bone-interface by the use of cadaver testings or finite element models [26].

Our in vitro data suggest the possibility of even an isolated use of the SIAF for fractures where external fixation alone would be considered adequate.

This would provide a quick intervention with early and simplified mobilization of the patient. It is known that, in the treatment of pelvic ring injuries, early mobilization significantly reduces posttraumatic complications and morbidity [5]. In fractures with intact posterior sacroiliac and sacro-tuberous ligaments (like LC II and APC II), posterior stabilization has to be postponed in some cases. By providing higher stability and lower infection and loosening rates [11-13], isolated preliminary anterior fixation with SIAF might be an alternative to external fixation. However, the promising results of this study have yet to be proven in vivo.

In this context, it is important to mention that SIAF seems to be associated with irritation of the lateral femoral cutaneous nerve in up to $27 \%$ of cases and with heterotopic ossifications in up to $32 \%$ [11-13]. In addition, components of SIAF are close to important anatomic structures, like the femoral vascular bundle and the urinary bladder [27].

Hence, careful adherence to the surgical anatomy is crucial.

\section{Conclusions}

In comparison with the standard external fixation system, the tested device for subcutaneous internal anterior fixation (SIAF) in vitro has similar translational and 
superior rotational stiffness. This technique might provide a useful tool in the treatment of pelvic ring injuries, especially in case of patients with concomitant abdominal injuries or risk factors for pin tract infection.

\section{Competing interests}

The authors declare that they have no competing interests.

\section{Authors' contributions}

GO, ST, SF, and CW participated in the study's design and planning. GO, ST, SF, GS, G-LS, and CW carried out the testing. GO, SF, and MS performed the data analysis. GO, SF, GW, H-PS, and CW drafted/revised the final manuscript. All authors read and approved the final manuscript.

\section{Acknowledgements}

We would like to thank Stryker (Kalamazoo, MI, USA) and Medtronic (Minneapolis, MN, USA) for donating the implants that were used for this study.

\section{Author details}

'Division of Trauma Surgery, University of Zurich, Raemistrasse 100, Zurich 8091, Switzerland. ${ }^{2}$ Institute for Biomechanics, ETH Zurich, HCI-E355.2 Wolfgang-Pauli-Strasse 10, Zurich 8093, Switzerland.

Received: 29 April 2013 Accepted: 28 March 2014

Published: 31 March 2014

\section{References}

1. Burgess AR, Eastridge BJ, Young JW, Ellison TS, Ellison PSJ, Poka A, Bathon $\mathrm{GH}$, Brumback RJ: Pelvic ring disruptions: effective classification system and treatment protocols. J Trauma 1990, 30(7):848-856.

2. Miller PR, Moore PS, Mansell E, Meredith JW, Chang MC: External fixation or arteriogram in bleeding pelvic fracture: initial therapy guided by markers of arterial hemorrhage. J Trauma 2003, 54(3):437-443.

3. Grimm MR, Vrahas MS, Thomas KA: Pressure-volume characteristics of the intact and disrupted pelvic retroperitoneum. J Trauma 1998, 44(3):454-459.

4. Gardner MJ, Kendoff D, Ostermeier S, Citak M, Hufner T, Krettek C, Nork SE: Sacroiliac joint compression using an anterior pelvic compressor: a mechanical study in synthetic bone. J Orthop Trauma 2007, 21(7):435-441.

5. Riemer BL, Butterfield SL, Diamond DL, Young JC, Raves JJ, Cottington E, Kislan K: Acute mortality associated with injuries to the pelvic ring: the role of early patient mobilization and external fixation. J Trauma 1993, 35(5):671-675. discussion 676-677.

6. Kim WY, Hearn TC, Seleem O, Mahalingam E, Stephen D, Tile M: Effect of pin location on stability of pelvic external fixation. Clin Orthop Relat Res 1999, 361:237-244.

7. Pohlemann T, Krettek C, Hoffmann R, Culemann U, Gansslen A: Biomechanical comparison of various emergency stabilization measures of the pelvic ring. Unfallchirurg 1994, 97(10):503-510.

8. Osterhoff G, Ossendorf C, Wanner GA, Simmen HP, Werner CM: Posterior screw fixation in rotationally unstable pelvic ring injuries. Injury 2011, 42(10):992-996.

9. Wanner GA, Keel M, Steckholzer U, Beier W, Stocker R, Ertel W: Relationship between procalcitonin plasma levels and severity of injury, sepsis, organ failure, and mortality in injured patients. Crit Care Med 2000, 28(4):950-957.

10. Mason WT, Khan SN, James CL, Chesser TJ, Ward AJ: Complications of temporary and definitive external fixation of pelvic ring injuries. Injury 2005, 36(5):599-604.

11. Kuttner M, Klaiber A, Lorenz T, Fuchtmeier B, Neugebauer R: The pelvic subcutaneous cross-over internal fixator. Unfallchirurg 2009, 112(7):661-669.

12. Vaidya R, Kubiak EN, Bergin PF, Dombroski DG, Critchlow RJ, Sethi A, Starr AJ: Complications of anterior subcutaneous internal fixation for unstable pelvis fractures: a multicenter study. Clin Orthop Relat Res 2012, 470(8):2124-2131.

13. Gardner MJ, Mehta S, Mirza A, Ricci WM: Anterior pelvic reduction and fixation using a subcutaneous internal fixator. J Orthop Trauma 2011, 26(5):314-321.
14. Dupont WD, Plummer WD: Power and Sample Size Calculations for studies Involving Linear Regression. Controlled Clinical Trials 1998, 19:589-601.

15. Vigdorchik JM, Esquivel AO, Jin X, Yang KH, Onwudiwe NA, Vaidya R: Biomechanical stability of a supra-acetabular pedicle screw Internal Fixation device (INFIX) vs External Fixation and plates for vertically unstable pelvic fractures. J Orthop Surg Res 2012, 7(1):31.

16. Moroz TK, Finlay JB, Rorabeck CH, Bourne RB: External skeletal fixation: choosing a system based on biomechanical stability. J Orthop Trauma 1988, 2(4):284-296.

17. Papathanasopoulos A, Tzioupis C, Giannoudis VP, Roberts C, Giannoudis PV Biomechanical aspects of pelvic ring reconstruction techniques: Evidence today. Injury 2010, 41(12):1220-1227.

18. Ponsen KJ, Hoek van Dijke GA, Joosse P, Snijders CJ: External fixators for pelvic fractures: comparison of the stiffness of current systems. Acta Orthop Scand 2003, 74(2):165-171.

19. Rieger $H$, Winckler S, Wetterkamp D, Overbeck J: Clinical and biomechanical aspects of external fixation of the pelvis. Clin Biomech (Bristol, Avon) 1996, 11(6):322-327.

20. van den Bosch EW, van Zwienen CM, van Dijke GA H, Snijders CJ, van Vugt AB: Sacroiliac screw fixation for tile B fractures. J Trauma 2003, 55(5):962-965.

21. Wang M, Dumas GA: Mechanical behavior of the female sacroiliac joint and influence of the anterior and posterior sacroiliac ligaments under sagittal loads. Clin Biomech (Bristol, Avon) 1998, 13(4-5):293-299.

22. Bergmann G, Graichen F, Rohlmann A, Bender A, Heinlein B, Duda GN, Heller MO, Morlock MM: Realistic loads for testing hip implants. Biomed Mater Eng 2010, 20(2):65-75.

23. Goel VK, Valliappan S, Svensson NL: Stresses in the normal pelvis. Comput Biol Med 1978, 8(2):91-104

24. Ferguson SJ, Winkler F, Nolte LP: Anterior fixation in the osteoporotic spine: cut-out and pullout characteristics of implants. Eur Spine J 2002, 11(6):527-534.

25. Lill H, Hepp P, Korner J, Kassi JP, Verheyden AP, Josten C, Duda GN: Proximal humeral fractures: how stiff should an implant be? A comparative mechanical study with new implants in human specimens. Arch Orthop Trauma Surg 2003, 123(2-3):74-81.

26. Bohme J, Shim V, Hoch A, Mutze M, Muller C, Josten C: Clinical implementation of finite element models in pelvic ring surgery for prediction of implant behavior: a case report. Clin Biomech (Bristol, Avon) 2012, 27(9):872-878.

27. Merriman DJ, Ricci WM, McAndrew CM, Gardner MJ: Is application of an internal anterior pelvic fixator anatomically feasible? Clin Orthop Relat Res 2012, 470(8):2111-2115.

doi:10.1186/1471-2474-15-111

Cite this article as: Osterhoff et al:: Mechanical testing of a device for subcutaneous internal anterior pelvic ring fixation versus external pelvic ring fixation. BMC Musculoskeletal Disorders 2014 15:111.

\section{Submit your next manuscript to BioMed Central and take full advantage of:}

- Convenient online submission

- Thorough peer review

- No space constraints or color figure charges

- Immediate publication on acceptance

- Inclusion in PubMed, CAS, Scopus and Google Scholar

- Research which is freely available for redistribution 